\title{
Gangrena de Fournier en el Hospital Escuela Universitario de Tegucigalpa, Honduras
}

\author{
Fournier's gangrene at the Hospital Escuela Universitario of \\ Tegucigalpa, Honduras \\ Daniel Martín Barahona-López, * Juan Luis Laínez-Mejía, * Luis Enrique Sánchez-Sierra, * \\ Carlos Felipe Matute-Martínez, ${ }^{*}$ Rafael Perdomo-Vaquero**
}

\begin{abstract}
Palabras clave: Gangrena de Fournier, síndrome de Fournier, fascitis necrotizante.

Key words: Fournier's gangrene, Fournier's syndrome, necrotizing fasciitis.
\end{abstract}

\section{RESUMEN}

La gangrena de Fournier es una fascitis necrotizante de origen polimicrobiano que afecta fascia y tejidos subcutáneos de las regiones genital, perineal y perianal; conlleva un riesgo elevado de morbimortalidad debido a la rápida diseminación del proceso. Objetivo: Contribuir al conocimiento de esta patología al establecer una caracterización clínica de los pacientes atendidos en el Hospital Escuela Universitario. Material y métodos: Estudio observacional, descriptivo y retrospectivo. Se estudiaron 40 casos de individuos entre los años 2010-2015, se valoraron datos epidemiológicos, clínicos y tratamiento. Resultados: De 40 casos, 37 fueron del sexo masculino y tres del femenino, con una relación de 12:1. La media de edad fue 51 años, con mayor incidencia en la quinta y sexta décadas de vida. Las principales manifestaciones clínicas fueron dolor, en $90.0 \%$ de los sujetos, fiebre, en $67.5 \%$, y secreción purulenta, en $55.0 \%$. El principal factor relacionado fue diabetes mellitus, presente en $37.5 \%$ de los enfermos. Proteus mirabilis fue el microorganismo aislado con mayor frecuencia de los cultivos de secreción, seguido de Escherichia coli. Conclusión: Aunque esta patología es poco frecuente, las complicaciones y secuelas son severas, por lo que se deben establecer un diagnóstico y tratamiento tempranos para controlar su rápida evolución.

\section{ABSTRACT}

Fournier's gangrene is a necrotizing fasciitis of polymicrobial origin that affects fascia and subcutaneous tissues of the genital and perianal regions; this condition carries a high risk of morbidity and mortality due to the rapid spread that the process can have. Objective: To contribute to the knowledge of this disease by establishing a clinical characterization of patients treated at the University Hospital. Material and methods: An observational, descriptive, retrospective study of 40 cases that were reviewed between 2010 and 2015; their epidemiological, clinical and treatment data were evaluated. Results: Of the 40 people who were included, 37 were male and three female, establishing a ratio of 12:1. The average age was 51 years; we found increased incidence in the fifth and sixth decades of life. The main clinical manifestation was pain, in the $90.0 \%$ of the patients, followed by fever, in $67.5 \%$, and purulent discharge, in $55.0 \%$; the main related factor was diabetes mellitus, present in $37.5 \%$ of the participants. Proteus mirabilis was the most frequently isolated organism in crop secretion, followed by Escherichia coli. Conclusions: Although this condition is rare, complications and sequelae in patients are severe, so an early diagnosis and treatment should be established to reduce the rapid progress it can present.
* Octavo año de la Carrera de Medicina y Cirugía. Médico en Servicio Social. ** Docente titular de la Facultad de Ciencias MédicasUNAH. Coordinador de Desarrollo Curricular y Área Básica de la Carrera de Medicina y Cirugía.

Hospital Escuela Universitario, Municipio del Distrito Central, Honduras.

Recibido: 19/05/2016 Aceptado: 25/11/2016

\section{INTRODUCCIÓN}

$\mathrm{L}$ a gangrena de Fournier es una infección rápidamente progresiva de la piel y tejidos blandos; ${ }^{1,2}$ usualmente es de etiología polimicrobiana y afecta, sobre todo, flora bacteriana de las zonas perineal y perianal. Con frecuencia se encuentra más de un organismo en los cultivos realizados: en promedio se aíslan tres bacterias por cultivo. ${ }^{3,4}$ La primera descripción de esta entidad fue por Baurianne en 1764. ${ }^{5}$
Presenta una incidencia de 1:7,500, con factores de riesgo asociados como diabetes mellitus, tabaquismo e hipertensión arterial. Realizar el diagnóstico temprano es importante, esto basándose en los criterios de Cymec para fascitis necrotizante: dolor severo, eritema, edema, linfadenopatías ausentes y cambios de coloración en la piel. ${ }^{6-8}$ Los resultados de laboratorio son sugestivos, pero no específicos; habitualmente se encuentra leucocitosis con presencia de células en bandas, lactato, pro- 
teína $\mathrm{C}$ reactiva y creatinina elevados; anemia y trombocitopenia atribuidas al proceso. . $^{3,9,10}$

El tratamiento quirúrgico debe ser oportuno, ya que la enfermedad puede avanzar en promedio una pulgada por cada hora transcurrida. Este tratamiento consiste en realizar limpieza quirúrgica de emergencia y brindar cobertura antibiótica de amplio espectro. ${ }^{11-13}$ En 1995, Eliahu Laor propuso valorar el pronóstico de mortalidad de los pacientes diagnosticados con gangrena de Fournier basado en criterios clínicos y estudios de laboratorio según su resultado al ingreso del individuo. ${ }^{14,15}$

El objetivo de este estudio fue caracterizar los sujetos con gangrena de Fournier que ingresaron al Hospital Escuela Universitario entre enero de 2010 y diciembre de 2015, lo que permitió obtener los principales factores predisponentes para el desarrollo de la enfermedad y las implicaciones que conlleva la progresión de la misma.

\section{MATERIAL Y MÉTODOS}

Tipo de estudio: Observacional, descriptivo y retrospectivo.

Muestra: Durante el periodo comprendido del 1 enero de 2010 al 31 de diciembre de 2015 (seis años) en el Hospital Escuela Universitario, se ingresó un total de 111 personas con el diagnóstico de fascitis necrotizante de Fournier. La revisión de los expedientes clínicos se llevó a cabo con el total de ingresos hospitalarios durante el periodo mencionado; de éstos se excluyeron 71 expedientes por no llenar los criterios de inclusión, con lo que se obtuvo un total de 40 expedientes clínicos.

Lugar de estudio: Hospital Escuela Universitario de Tegucigalpa, Honduras. Éste es un centro de referencia a nivel nacional, principalmente para las zonas centro, sur y oriente del país. Previamente a la recolección de datos, se obtuvo la autorización por parte de la Coordinación de la Carrera de Medicina y Cirugía de la Facultad de Ciencias Médicas de la Universidad Nacional Autónoma de Honduras. Los criterios de inclusión al estudio fueron: 1) paciente con diagnóstico de gangrena de Fournier, 2) individuos mayores de 18 años, 3) expedientes disponibles en el Departamento de Archivo. Los criterios de exclusión al estu- dio fueron: 1) expediente no disponible en el Departamento de Archivo, 2) sujetos menores de 18 años y 3) personas con diagnóstico de fascitis necrotizante que no fuera de origen genitourinario, perineal $y / o$ perianal.

La recolección de datos se llevó a cabo mediante la aplicación de una encuesta completada por los investigadores a partir de los expedientes clínicos de los pacientes; ésta consistía en un cuestionario de 50 preguntas, 32 abiertas y 18 cerradas. Se recolectaron datos generales, sociodemográficos, antecedentes médicos del individuo y familiares, tiempo de evolución; se obtuvieron datos sobre las intervenciones quirúrgicas y los estudios de laboratorio realizados a cada sujeto.

Aspectos bioéticos: se aseguró la confidencialidad y no divulgación de los datos personales de cada uno de los expedientes clínicos de los enfermos, evitando cualquier tipo de daño a su integridad como seres humanos o sus derechos como pacientes.

Se realizó el análisis descriptivo de las variables cuantitativas y cualitativas de los resultados del grupo de trabajo, se calcularon las medias, frecuencias, varianzas y valor $p$ en función de determinar valores normales y tendencias de las variables. La matriz de datos fue construida en el paquete de Microsoft Office Excel $2016^{\circledR}$ y el análisis estadístico se llevó a cabo con el paquete Epi-Info versión 7.1.5.2; se estableció una significancia estadística para valores de $\mathrm{p}$ $<0.05$.

\section{RESULTADOS}

De una muestra de individuos consecutivos durante el periodo de estudio de enero de 2010 a diciembre de 2015 se ingresaron 111 casos con diagnóstico de gangrena de Fournier; de éstos, se eliminaron 71 por no llenar los criterios de inclusión. La muestra estudiada fue de 40 sujetos con diagnóstico de gangrena de Fournier.

La frecuencia de casos por año se representó de la siguiente manera: tres (7.5\%) reportados en el año 2010, cuatro (10\%) en el año 2011, nueve (22.5\%) en el año 2012, seis (15\%) en el año 2013; el pico máximo fue de 14 (35\%) casos reportados en el año 2014; en 2015 se reportaron cuatro (10\%) (Figura 1). 


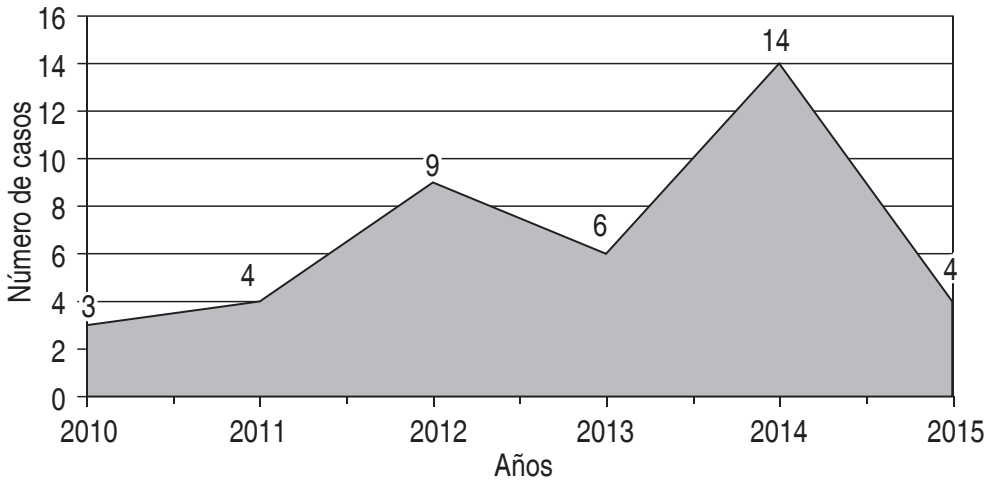

Figura 1. Incidencia de la patología por año.

De los 40 casos $(n=40)$ estudiados, el rango de edad fue de 19 hasta 93 años, con una edad promedio de 51 años, siendo los grupos etarios más afectados los comprendidos en la quinta y sexta décadas de vida, con $10(25.0 \%)$ y ocho (20.0\%) casos, respectivamente. Del total de pacientes, $37(92.5 \%)$ fueron del sexo masculino y tres $(7.5 \%)$, del sexo femenino, obteniendo una relación 12:1. Los signos y síntomas iniciales más frecuentes en las personas con gangrena de Fournier fueron dolor en 36 (90\%) casos, fiebre en $27(67.50 \%)$, salida de secreción purulenta en 22 (55\%); cinco (12.5\%) de ellos manifestaron iniciar con una masa (Cuadro I).

El promedio de días transcurridos desde el comienzo de los síntomas hasta el día de la primera atención en el hospital fue de 14, mientras que el origen del proceso fue genitourinario en $23(57.50 \%)$ de los enfermos y anorrectal en 17 (42.50\%) casos. Las comorbilidades y hábitos tóxicos más frecuentes fueron diabetes mellitus en 15 (37.5\%) pacientes, alcoholismo en 13 (32.5\%), hipertensión arterial en nueve (22.5\%), tabaquismo en ocho $(20.0 \%)$ y cirugía previa en seis $(15.0 \%)$ de los individuos (Figura 2).

Los sujetos permanecieron un promedio de 34.4 días dentro del centro hospitalario; se realizaron 150 intervenciones quirúrgicas. El promedio de visitas a quirófano por persona fue de 3.7; de éstas, las intervenciones más realizadas fueron limpiezas y desbridamientos quirúrgicos (91 intervenciones), colostomías (15), cistostomías (10) y orquiectomía (en 6 ocasiones) (Figura 3).

Se obtuvo crecimiento microbiológico en 10 cultivos de secreción; de éstos, en cinco se observó Proteus mirabilis, en cuatro Esche- richia coli, en dos Klebsiella pneumoniae. Para Candida no albicans, Morganella morganii, Staphylococcus aureus, Pseudomona aeruginosa y Staphylococcus haemolyticus se obtuvo crecimiento en una ocasión cada uno (Figura 4). Los antimicrobianos más usados fueron metronidazol, en 27 (67.5\%) enfermos, ceftriaxona, en $20(50 \%)$, piperacilina/tazobactam, en 14 (35\%), y clindamicina, en $12(30 \%)$ pacientes.

\section{DISCUSIÓN}

La gangrena de Fournier es una fascitis necrotizante que afecta la región perineal y perianal. A pesar de no ser una patología común, presenta un alto riesgo de morbimortalidad. ${ }^{3,16}$ La clínica de la patología es variable; se puede encontrar desde sólo un dolor localizado hasta necrosis rápidamente progresiva de la zona o sepsis generalizada; aunque usualmente comienza como un cuadro febril de pocos días de duración con dolor en región perineal y/o perianal, es posible observar inflamación o eritema localizado o difuso con salida de secreción purulenta. En individuos con neuropatía diabética puede haber ausencia de dolor durante la fase inicial. 3,5,8 Cuando existe infección por bacterias formadoras de gas, se puede encontrar enfisema subcutáneo con extensión a la pared abdominal o tejidos del miembro inferior, que se manifiesta por la presencia de crepitación de tejidos blandos a la palpación. ${ }^{8,17}$

En nuestro estudio, la mayoría de los sujetos afectados pertenecían al género masculino, obte-

Cuadro I. Síntomas y signos más frecuentes.

\begin{tabular}{lrr} 
Síntomas y signos & Frecuencia & Porcentaje \\
\hline Dolor & 36 & 90.0 \\
Fiebre & 27 & 67.5 \\
Secreción purulenta & 22 & 55.0 \\
Masa & 5 & 12.5 \\
Edema & 4 & 10.0 \\
Necrosis & 3 & 7.5 \\
Eritema & 1 & 2.5 \\
Alteración de la conciencia & 1 & 2.5 \\
Enfisema subcutáneo & 1 & 2.5 \\
Escalofríos & 1 & 2.5
\end{tabular}




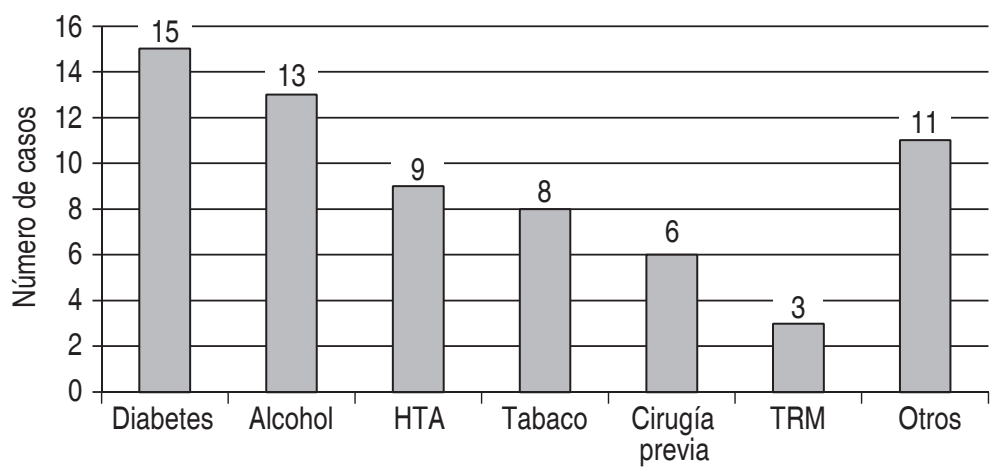

HTA = Hipertensión arterial; TRM = Trauma raquimedular.

Figura 2. Factores de riesgo más frecuentes.

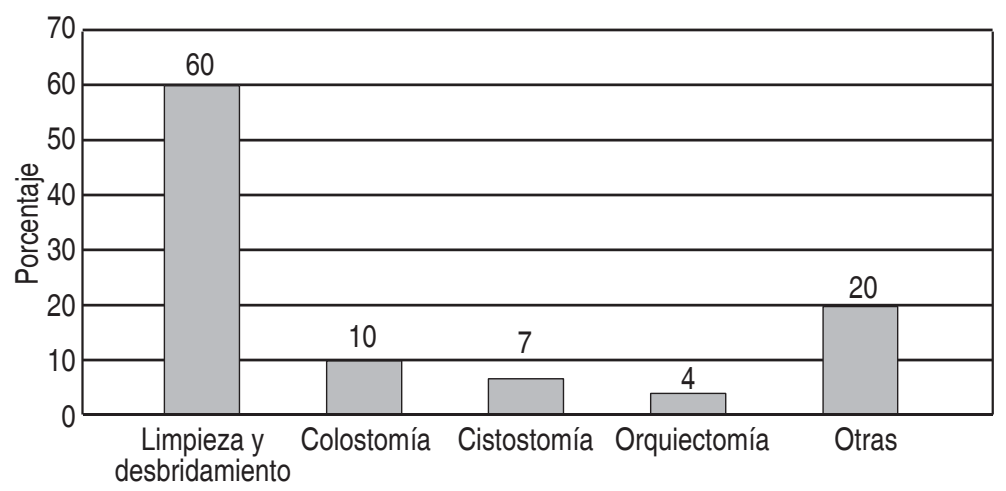

Figura 3. Intervenciones quirúrgicas más frecuentes.

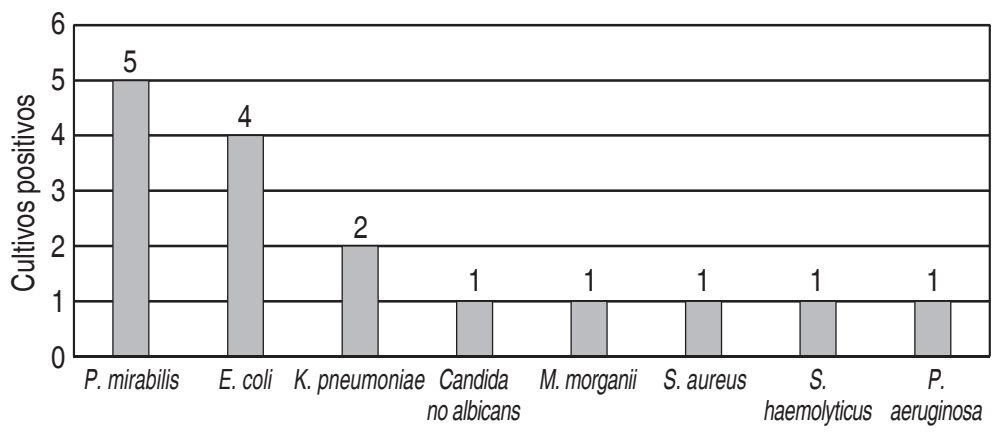

Figura 4. Microorganismos más reportados en cultivos de secreción.

niendo una relación de 12:1, similar a la relación 10:1 mencionada en otros trabajos; 4,18 la edad promedio encontrada fue 51 años, cercana a la media de 52 años de la literatura consultada. ${ }^{7}$

Dentro de los factores relacionados con el desarrollo de esta patología destaca la diabetes mellitus (presente en el $40-60 \%$ de las perso- nas); otros que pueden estar presentes son alcoholismo, hipertensión arterial sistémica, obesidad, insuficiencia renal, insuficiencia hepática, tabaquismo, neoplasias, procedimientos quirúrgicos recientes, inmunosupresión, tratamiento de quimioterapia y radioterapia. ${ }^{19,20} \mathrm{En}$ nuestra investigación se halló que $37.5 \%$ de los enfermos padecían diabetes mellitus, siendo la principal comorbilidad encontrada.

La diseminación de la patología se ve favorecida por la continuidad de los planos anatómicos a través de las fascias superficiales de Colles, Scarpa y Dartos, lo que también puede favorecer su invasión a planos más profundos, como la fascia profunda de Buck y la cavidad abdominal; incluso puede abarcar las regiones inguinal y femoral. ${ }^{19,21}$ La progresión de la enfermedad produce endarteritis obliterante, ocasionando la formación de trombosis en los vasos sanguíneos; esto provoca hipoxia tisular y facilita la colonización bacteriana. Esta proliferación promueve la producción de endotoxinas y exotoxinas, que al llegar a la circulación sistémica desencadenan un síndrome de respuesta inflamatoria sistémica (SIRS) que puede progresar a shock séptico y síndrome de disfunción multiorgánica. ${ }^{5,19-22}$

El principal microorganismo asociado a esta patología es la Escherichia coli; otros que están relacionados son Bacteroides, Streptococcus pyogenes, Staphylococcus aureus, Clostridium, Pseudomonas aeruginosa, Klebsiella, Proteus y Enterococos. ${ }^{3,4,6}$ El principal agente aislado en nuestro estudio fue Proteus mirabilis.

El síntoma más común en los pacientes incluidos en este trabajo fue el dolor en la región genital y/o perianal, reportado en $90 \%$ de los casos. Se sabe que la presentación del síndrome puede ser bastante inespecífica; los individuos usualmente comienzan con un cuadro febril de pocos días de duración, dolor en la región perineal y/o perianal. Al examen físico, se puede observar inflamación o eritema de forma localizada o difusa, con salida de secreción purulenta. En algunos casos, en sujetos diabéticos puede haber ausencia de dolor desde la fase inicial de la enfermedad debido a neuropatía diabética avanzada. $3,5,8$

El tratamiento inicial consiste en realizar limpieza quirúrgica junto a un manejo an- 
tibiótico de amplio espectro para gérmenes Gram negativos, Gram positivos y anaerobios. Al obtener resultados de cultivos positivos se debe cambiar la cobertura antibiótica a la que se reporten sensibles los microorganismos aislados. Se debe disponer de un cupo en la Unidad de Terapia Intensiva previendo la falta de respuesta o complicaciones de la enfermedad..$^{13}$ Recientemente, nuevas terapias como la oxigenoterapia y la aplicación de factores de crecimiento han mostrado mejoría en los casos utilizados. ${ }^{23,24}$

En nuestra investigación, la media de la estancia intrahospitalaria fue 34.4 días, cercana al promedio de 36 días de manejo intrahospitalario. ${ }^{5}$ Durante este tiempo, se realizó un aproximado de 3.7 intervenciones quirúrgicas por persona, muy similar al promedio de 3.3 encontrado; ${ }^{25}$ las más frecuentemente realizadas fueron limpiezas quirúrgicas, colostomías y cistostomías. Debido a las secuelas que conlleva la gangrena de Fournier, tanto por la progresión del proceso infeccioso como por su tratamiento quirúrgi$\mathrm{CO}$, se debe estar al tanto de las alteraciones que se puedan presentar durante la estancia hospitalaria y tras el egreso, ya que algunas de éstas pueden repercutir de manera permanente en el enfermo. ${ }^{26-28}$

La mortalidad en nuestro estudio fue del $0 \%$. Encontramos que dentro de esta patología este dato es muy variable, reportándose series desde $0 \%$ hasta estudios con una mortalidad del $100 \%$, con un rango promedio de mortalidad de 3 a $67 \%{ }^{29,30}$

\section{CONCLUSIÓN}

La gangrena de Fournier es un proceso polimicrobiano al que están relacionadas diversas condiciones patológicas, especialmente la diabetes mellitus, la cual es considerada como el principal factor de riesgo para el desarrollo de la entidad.

Su tratamiento debe ser combinado entre un manejo quirúrgico oportuno y una cobertura antibiótica adecuada; sin embargo, incluso con un tratamiento adecuado, esta patología puede conllevar una larga estancia hospitalaria, con las implicaciones que esto representa para el paciente.
La rápida evolución de la enfermedad hace que el diagnóstico temprano sea de gran importancia para establecer el tratamiento oportuno y reducir el riesgo de complicaciones.

\section{REFERENCIAS}

1. Parra CP, Pérez ES, Patiño RM, Castañeda SS, García VJ. Actualización en fascitis necrotizante. Semin Fund Esp Reumatol. 2012; 13: 41-48.

2. Puerto PA, García SE, Morilla GM, Rodríguez RM, Bravo SC, Urbina PAP, et al. Gangrena de Fournier: análisis descriptivo de nuestra serie de 26 pacientes. Rev Arg de Urol. 2013; 78: 90-94.

3. Trejos-Mazariegos S, Torrealba Alonso M. Gangrena de Fournier. Revista Médica de Costa Rica y Centroamérica LXX [seriada en línea]. 2013 [citado 11 de marzo de 2016]; (606): [7 páginas].

4. Cancino BC, Avendaño HR, Poblete AC, Guerra HK. Gangrena de Fournier. Cuad Cir. 2010; 24 (1): 28-33.

5. Navarro-Vera JA. Gangrena de Fournier. Evid Med Invest Salud. 2010; 3: 51-57.

6. Méndez-Rosales M, Aymaya GC, Gutiérrez CF, Huacota SM. Gangrena de Fournier pos-prostatectomía transuretral por cáncer de próstata: a propósito de un caso. Rev Med-Cient. 2011; 2: 64-67.

7. Castillo HF, Moraga CJ, Pérez CP, Álvarez ZC, Iglesias BA. Diagnóstico y manejo precoz de la gangrena de Fournier. Rev Chil Cir. 2015; 67: 181-184.

8. Blanco F, Caradonti M, Rivero D, Iborra F, Franceschelli A, Smolje L. Fascitis necrotizante perineal: gangrena de Fournier. Hosp Aeronaut Cent. 2014; 9: 113-120.

9. Chennansetty A, Khourdaji I, Burks F, Killinger KA. Contemporary diagnosis and management of Fournier's gangrene. Thev Adv Urol. 2015; 7: 203-215.

10. López BM, Alba CJ, Guerrero AG, Améndola MA, Casillas MJ. La gangrena de Fournier: evaluación con tomografía computada. Anales de Radiología México. 2012; 12: 98-103.

11. Akilov O, Pompeo A, Sehrt D, Bowlin P, Molina WR, Kim FJ. Early scrotal approximation after hemiscrotectomy in patients with Fournier's gangrene prevents scrotal reconstruction with skin graft. Can Urol Assoc J. 2013; 7: E481-485.

12. Thawaini A, Khan A, Malik A, Cherian J, Barua J, Shergill I, et al. Fournier's gangrene and its emergency management. Postgrad Med J. 2006; 82: 516-519.

13. Mallikarjuna MN, Vijayakumar A, Patil VS, Shivswamy BS. Fournier's gangrene: current practices. ISRN Surgery [seriada en línea]. 2012 [citado 13 de marzo de 2016]; [8 páginas]. Disponible en: http://www. hindawi.com/journals/isrn/2012/942437/cta/

14. García MA, Turégano FF, Cuadrado AM, Andueza LJ, Cano BJ, Pérez LM. Factores predictivos de mortalidad en la gangrena de Fournier: serie de 59 casos. Cir Esp. 2015; 93: 12-17.

15. Laor E, Palmer LS, Tolia BM, Reid RE, Winter HI. Outcome predictions in patients with Fournier's gangrene. J Urol. 1995; 154: 89-92.

16. Jimenez-Pacheco A, Arrabal-Polo MA, Arias-Santiago S, Arrabal-Martin M, Nogueras-Ocaña M, ZuluagaGomez A. Fournier Gangrene: description of 37 cases 
and analysis of associated health care costs. Actas Dermosifiliogr. 2012; 103: 29-35.

17. Urdaneta CE, Méndez PA, Urdaneta CA. Gangrena de Fournier. Perspectivas actuales. An Med Interna. 2007; 24: 190-194.

18. Sedano-Basilio JE, Cornejo-Dávila V, Trujillo-Ortiz L, Cantellano-Orozco M, Fernández-Noyola G, MartínezArroyo C, et al. Experiencia y revisión de la literatura en el manejo de gangrena de Fournier en una institución 2008-2015. Rev Mex Urol. 2016; 76: 29-35.

19. Mehl AA, Nogueira FD, Marques LM, Mamprim MM, Berger R, Krauss D, et al. Management of Fournier's Gangrene: experience of a university hospital of $\mathrm{Cu}$ ritiba. Rev Col Bras Cir. 2010; 37: 435-441.

20. Montoya CR, Izquierdo ME, Pietricica BN, Pellicer FE, Aguayo AJ, Miñana LB. Gangrena de Fournier. Análisis descriptivo de 20 casos y revisión de la bibliografía científica. Actas Urol Esp. 2009; 33: 873-880.

21. Etcheverry MG, Plerini A, Brosutti O, G Ruiz, Canga V. Manejo de la Gangrena de Fournier: nuestra experiencia. RevArg Res Cir. 2015; 20: 19-21.

22. Rodríguez VA, Larios GC, Garcia CG, Rodríguez SI, López PJ. Gangrena de Fournier. Rev Medica Sanitas. 2015; 18: 212-219.

23. Ming-Chan $\mathrm{H}$, Chia-Lin $\mathrm{C}$, Li-Chin $\mathrm{C}$, Chung-Han $\mathrm{H}$, Ko-Chi N, Hsien-Lin C, et al. The role of hyperbaric oxygen therapy in treating extensive Fournier's gangrene. Urological Science [seriada en línea]. 2015 [citado 13 de marzo de 2016]; [6 páginas]. Disponible en: http://www.urol-sci.com/article/S18795226(15)00396-6/pdf

24. González SG, Díaz MJ, Collera RS, González GG, Romero RJ, Rivero OJ. Gangrena de Fournier. Rev Cubana Cir [seriada en línea]. 2010 [citado 13 de marzo de 2016]; 49(3): [6 páginas]. Disponible en: http://scielo. sld.cu/pdf/cir/v49n3/cir09310.pdf
25. Azolas M R. Factores de riesgo para mortalidad en gangrena de Fournier. Rev Chil Cir. 2011; 63(3): 270275.

26. Ballesteros JR, Garcia-Tarriño R, Ríos M, Domingo A, Rodríguez-Roiz JM, Llusa-Pérez $M$, et al. Necrotizing soft tissue infections: a review. International Journal of Advanced Joint Reconstruction [seriada en línea]. 2016 [citado 13 de marzo de 2016]; 3 (1): [9 páginas]. Disponible en: http://www.healthyjoints.eu/wp-content/ uploads/2016/02/2016_IJAJR_Necrotizing-soft-tissueinfections-Review.pdf

27. Chlya PL, Igenge JZ, Mabula JB, Simbila S. Fournier's gangrene at a tertiary health facility in northwestern Tanzania: a single centre experiences with 84 patients. BMC Research notes [seriada en línea]. 2015 [citado 13 de marzo de 2016]; 8 (481): [7 páginas]. Disponible en: http://bmcresnotes.biomedcentral.com/ articles/10.1186/s13104-015-1493-1

28. Shashireka CA, Pramod T, Nagarj KN, Kumar H, Rakesh $\mathrm{N}$. Evaluation of Fournier's gangrene severity index in the management of Fournier's gangrene: retrospective study. Int Surg J. 2016; 3: 169-172.

29. García A, Turégano F, Martín J, Vaquero A, Camarero C, Pérez MD. Gangrena de Fournier: Análisis de una serie de 34 casos. Rev Colomb Cir. 2010; 25: 151-157.

30. Fuentes Valdés E, Núñez Roca A, Kapaxi Andrade J, Fernandes da Cunha Y. Gangrena de Fournier. Rev Cubana Cir. 2013; 52: 91-100.

Correspondencia:

Luis Enrique Sánchez-Sierra

Residencial La Granja, Casa 7, Bloque B, Municipio del Distrito Central, Honduras. Tel: 504-9947-2848

E-mail: luensasi90@hotmail.com 